\title{
IgG4-related disease in endocrine practice
}

\author{
Agata Rzepecka, Anna Babińska, Krzysztof Sworczak
}

Department of Endocrinology and Internal Medicine, Medical University of Gdansk, Gdansk, Poland

Submitted: 26 July 2017

Accepted: 28 September 2017

Arch Med Sci 2019; 15, 1: 55-64

DOI: https://doi.org/10.5114/aoms.2017.70889

Copyright @ 2017 Termedia \& Banach

\begin{abstract}
IgG4-related disease is a set of symptoms resulting from a chronic, usually multiple organ inflammatory condition which affects various organs. It consists of lymphoplasmacytic infiltrations with attendant fibrosis and deep vein thrombosis. Frequently observed tissue lesions are accompanied by elevated IgG4 levels in serum. The etiopathogenesis of the lesions is of multifactor character and the clinical manifestation of the disease is highly diverse. The diagnostic process is based on the patient's medical history, clinical examination and additional tests, including a histopathological examination of the infected organ's tissues. Almost forty different locations of the disease have been reported, including disorders of the endocrine system. IgG4-related endocrinopathies are quite rare. However, it is likely that the diagnosis is under-reported due to lack of awareness of this clinical entity. Despite increasing interest in the subject, there are not enough reliable studies evaluating the link between IgG4-RD and endocrine disorders.
\end{abstract}

Key words: IgG4-related disease, Hashimoto's thyroiditis, Riedel's thyroiditis, hypophysitis, Graves' ophthalmopathy.

\section{Introduction}

IgG4-related disease (IgG4-RD) is a set of signs and symptoms resulting from a chronic, usually multiple organ inflammatory condition which affects various tissues and organs. It consists of lymphoplasmacytic infiltrations with attendant fibrosis and deep vein thrombosis [1]. Frequently observed tissue lesions are accompanied by elevated IgG4 levels in serum. The etiopathogenesis of the lesions is of multifactor character and includes allergy mechanisms as well as infectious and autoimmune factors. Therefore, medical signs and diagnostics pose a serious challenge to clinicians. The epidemiology of IgG4-RD has not been fully clarified yet. The majority of cases are reported in Asia, though apparently IgG4-RD may occur all over the world [2]. According to Japanese researchers, the average number of newly diagnosed patients is $0.28-1.08 / 100000$ in the whole population [3]. In the medical literature different names have been employed to describe IgG4-RD. Only after a consensus had been reached by two independent Japanese research committees was the currently used name finally acknowledged [4]. The systemic character of IgG4-RD was first observed in 2003, mainly with reference to autoimmune pancreatitis [5]. Since then, almost forty different locations of the disease have been reported, including disorders of the endocrine system [2]. The IgG4-related endocrinopathies are quite rare, and thyroid involvement is
Corresponding author:

Agata Rzepecka MD

Department of

Endocrinology and

Internal Medicine

Medical University

of Gdansk

7 Debinki St

80-952 Gdansk, Poland Phone/fax: +48 695906153

E-mail: agatarzepecka@ gumed.edu.pl 
believed to occur in $4 \%$ to $6 \%$ of cases. However it is likely that the diagnosis is under-reported due to lack of awareness of this clinical entity. Despite an increasing interest in the subject, there are not enough reliable studies evaluating the link between IgG4-RD and endocrine disorders.

\section{Epidemiology}

Men are usually reported to suffer from IgG4-RD more frequently than women although in the case of involvement of head and neck organs these proportions become equal $[6,7]$. Mean age at the onset of the disease is between 50 and 60 years $[2,8]$; however, the disease may occur at any age, even in children $[9,10]$.

Most frequently, the lesions are located in organs of the digestive system such as the pancreas, bile ducts and salivary glands, as well as lacrimal glands, soft tissues of the orbital cavities and lymph nodes. Less frequently the disease may also be located in the mediastinum, respiratory system, retroperitoneal space, central nervous system, genitourinary system, mammary gland, prostate gland, cutis and blood vessels $[2,3]$. Among the most frequently infected endocrine glands are the thyroid and pituitary. Table I presents the most common organ locations in three large case series.

\section{Pathophysiology of IgG4}

The pathogenesis of IgG4-related disease is not fully elucidated. A combination of mechanisms of the innate and adaptive immune system, as well as genetic factors, contributes to the development of the disease. Most studies so far have examined only Asian patients with autoimmune pancreatitis (AIP), a pancreatic manifestation of IgG4-related disease.

\section{The role of IgG4}

Immunoglobulin G4 is one of the factors contributing to the development of IgG4-RD. However, its role in this complex process has not been fully explained yet. It has been established that in physiological conditions IgG4 constitutes the lowest IgG percentage in serum (3-6\%). It is characterized by unique properties, such as greater sensitivity to chemical factors as well as incapability of creating immune complexes $[11,12]$. It has been demonstrated that IgG4 production depends on T2 lymphocytes, which participate in allergization processes. An IgG4-related physiological response may also be triggered by a repetitive exposure to an antigen, e.g. in food allergy [13]. Although IgG4 does not have the ability to activate the classical complement pathway, it contributes to an inflammatory response through histamine release from basophils [14], which may partially account for its elevated levels in patients with atopic diseases and clinical improvement following the elimination of food allergens from the diet [15]. In the medical literature there are reports regarding the "inhibiting" character of the IgG4 antibody. Van der Giessen et al. observed a high concentration of this immunoglobulin following successful immunotherapy of IgE-related pollen allergies [16].

Table I. Comparison of organ involvement in three large case series

\begin{tabular}{|c|c|c|c|}
\hline Organ involvement & $\begin{array}{l}\text { Wallace } \text { et al. [6] } \\
\text { Percentage of } 125 \text { cases }\end{array}$ & $\begin{array}{l}\text { Inoue } \text { et al. }[82] \\
\text { Percentage of } 235 \text { cases }\end{array}$ & $\begin{array}{l}\text { Ebbo et al. }[83] \\
\text { Percentage of } 25 \text { cases }\end{array}$ \\
\hline Salivary gland & & 34 & 44 \\
\hline Submandibular glands & 28.0 & & \\
\hline Parotid glands & 16.8 & & \\
\hline Lacrimal gland & & 23 & 12 \\
\hline Pancreas & 19.2 & 60 & 52 \\
\hline Bile duct/biliary & 9.6 & 13 & 32 \\
\hline Orbital cavity & 22.4 & 4 & 4 \\
\hline Lungs & 17.6 & 13 & 12 \\
\hline Kidneys & 12.0 & 23 & 44 \\
\hline Aorta & 11.2 & 20 & 24 \\
\hline Lymph nodes & 27.2 & & 76 \\
\hline Intraperitoneal fibrosis & 18.4 & 4 & 32 \\
\hline Pituitary gland & & & 8 \\
\hline Thyroid & 5.6 & & \\
\hline
\end{tabular}


Elevated IgG4 levels may be observed not only in patients with IgG4-RD (in approximately $60-70 \%$ of patients), but also in a chronic inflammatory condition, infections, cancer, autoimmune diseases, vasculitis and drug hypersensitivity.

\section{Pathomechanism}

Several groups have tried to identify autoantigens based on the hypothesis that IgG4-related disease is an autoimmune disorder. The factor which triggers the disease process in IgG4-RD is still being searched for. Until now, a few antigens have been identified, including lactoferrin, carbonic anhydrase (CA-II), and pancreatic secretory trypsin inhibitor (PSTI) $[17,18]$. Recent publications suggest a possible role of prohibitin as an antigen which induces the generation of antibodies that contribute to the tissue growth and development of the IgG4-RD [19]. Others, on the other hand, indicate a possible role of molecular mimicry with the participation of Helicobacter pylori [20].

Increased tissue levels of Th2-cytokines including IL-4, IL-5, IL-10 and IL-13 were found in IgG4-RD. These cytokines also trigger antibody class switch to IgE and eosinophilic migration and infiltration. A role for elevated levels of regulatory T cells (Treg) has also been suggested. Two regulatory cytokines, IL-10 and transforming growth factor- $\beta$ (TGF- $\beta$ ), were also significantly overexpressed. Il-10 directs $B$ cells to produce IgG4, while TGF- $\beta$, a key pro-fibrotic cytokine, induces the deposition of extracellular matrix proteins and type 1 collagen, leading to fibroplasia. Further, $20 \%$ of IgG4-RD patients have allergic diseases (e.g. bronchial asthma or drug allergy), and tissue eosinophilia is typical [21]. However, Della Torre et al. stated that the majority of patients with IgG4-RD are nonatopic and that the prevalence of atopy in this disease is no higher than that expected in the general population [22]. A study showed that there are increased numbers of $M 2$ macrophages in submandibular glands affected by IgG4-RD and that fibrosis is associated with an increased number of $\mathrm{M} 2$ macrophages [23].

The $B$ cell compartment of patients with IgG4-RD has been studied extensively, because IgG4 elevation in the serum and the abundance of IgG4-plasma cells in the biopsies initially suggested an underlying lymphoproliferative condition. In B-cell development, plasmablast differentiation occurs between activated B cells and plasma cells. Wallace et al. reported that circulating plasmablasts are elevated in active IgG4-RD, even in patients with normal serum IgG4 concentrations, and that their levels reflect disease activity [24].

In the IgG4-RD pathomechanism, there have also been detected certain abnormalities in cell-mediated immunity. Monocyte and basophil disorders with the overproduction of cytokines such as BAFF and APRIL have been reported [25].

There is some evidence from Japanese studies that certain HLA alleles - particularly DRB ${ }^{\star} 0405$ and $\mathrm{DQB}^{\star} 0401$ - increase the susceptibility to type 1 autoimmune pancreatis (AIP). Furthermore, a few single-nucleotide polymorphisms (SNP) in genes related to the immune system such as CTLA-4, Fc-receptor-like 3 have been linked to IgG4-RD [21].

Figure 1 presents a simplified diagram of the IgG4-RD pathomechanism.

\section{Clinical manifestation}

Clinical manifestation of the disease is highly diverse, and its course in the initial stage is often asymptomatic. Therefore, the appearance of the symptoms seems to depend on the stage of the disease process, the number of infected organs and the duration of the disease. During the development of the disease, diffuse or focal organ enlargement may be observed, together with pseudotumours, erosion and sclerosis of adjoining bones [7]. In approximately $60 \%$ of patients at the moment of the diagnosis the disease process is already of multiorgan character. One third of the patients have a positive medical history for atopic diseases, which refers mostly to the respiratory system, cutis and soft tissues of orbital cavities [22]. General symptoms, such as fever, weight loss, and asthenia, are not frequently observed. Lymphadenopathy is reported in nearly $42 \%$ of patients with a systemic form of the disease. The appearance of specific symptoms depends on the location of the disease process and involves abdominal pain, keratoconjunctivitis sicca, various respiratory disorders, pruritus and diarrhea [26].

\section{Diagnostics and its criteria}

The diagnostic process is based on the patient's medical history, clinical examination and additional tests, including a histopathological examination of the infected organ's tissues [27]. Umehara et al. have established clinical criteria which determine the likelihood of IgG4-RD diagnosis (Table II) [4].

The microscopic image of the infected tissue is typically characterized by dense infiltrations formed by lymphoplasmacytic cells ( $B$ and $T$ lymphocytes), storiform fibrosis and thromboangiitis obliterans. Eosinophilic infiltrations are sometimes reported, especially in lesions located in orbital cavities or the upper respiratory tract [28].

Upon suspicion of IgG4-RD, it is necessary to perform immunohistopathological tests in order to identify lgG4+ cells and evaluate the lgG4+/ IgG ratio, which in the case of IgG4-RD diagnosis should be higher than $0.4[4,28]$. 

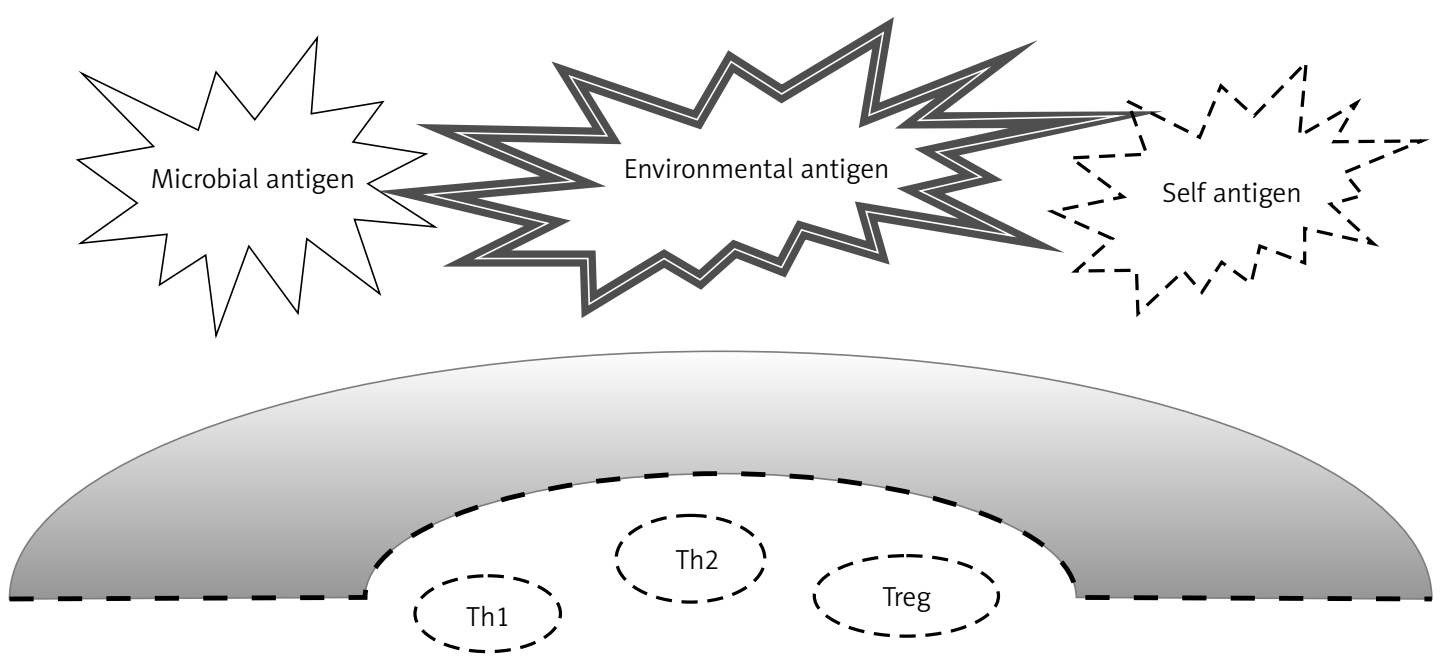

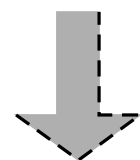

IL-4

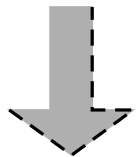

IL-13

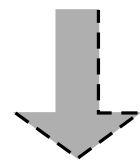

TGF- $\beta$
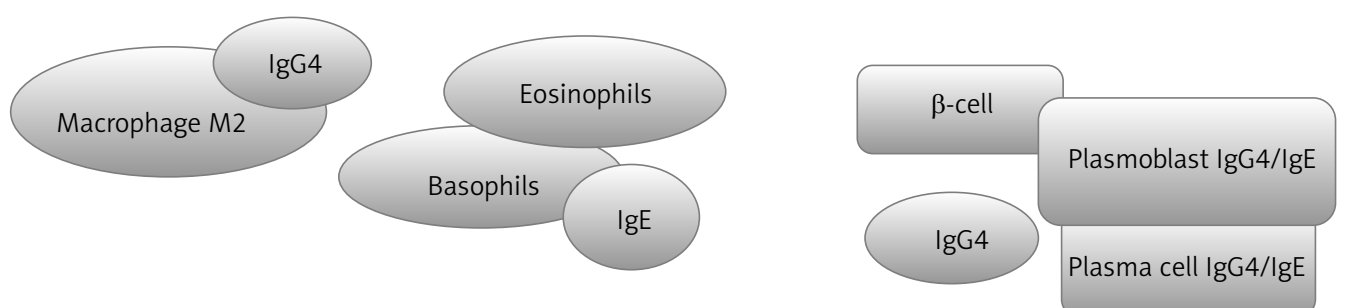

Storiform fibrosis

Figure 1. Diagram of the IgG4-RD pathomechanism

Table II. Criteria for the diagnosis of IgG4-RD according to Umehara et al. [4]

\begin{tabular}{|c|c|}
\hline 1 & $\begin{array}{l}\text { Characteristic symptoms of organ } \\
\text { infection (in the form of diffuse or } \\
\text { tumorous enlargement) or organ disorder }\end{array}$ \\
\hline 2 & Elevated IgG4 levels in serum (> $135 \mathrm{mg} / \mathrm{dl}$ ) \\
\hline 3 & $\begin{array}{l}\text { Lesions observed in histopathological tests: } \\
\text { - Lymphoplasmacytic infiltration and fibrosis } \\
\text { - Infiltration of IgG4-positive cells > } 10 \\
\text { IgG4-positive cells in the field of vision } \\
\text { in a high definition microscope and with } \\
\text { lgG4+/lgG ratio > 40\% }\end{array}$ \\
\hline \multicolumn{2}{|c|}{ Diagnosis of IgG4-related disease: } \\
\hline Certain & $1+2+3$ \\
\hline Probable & $1+3$ \\
\hline Likely & $1+2$ \\
\hline
\end{tabular}

So far it has been established that there may be a correlation between IgG4 concentration in serum and the activity of IgG4-RD. High levels of this immunoglobulin are usually associated with a more aggressive course of the disease, concurrence of IgG4-related lesions in other organs and probably a reduced response to the treatment [27]. What hinders the diagnostic process to a certain extent is the fact that IgG4 concentration may be within the reference range even in half of the patients with an active form of the disease confirmed in histopathological tests [6]. Therefore, isolated elevated IgG4 levels do not determine the diagnosis.

Abnormalities in laboratory tests are not very specific and include moderately increased inflammation markers (CRP in $25 \%$ of cases), mild eosin- 
ophilia (in 34\% of cases), and sporadically erythrocytopenia and thrombocytosis [18]. Furthermore, there are visibly elevated IgE levels [15], polyclonal hypergammaglobulinemia [29], decreased levels of complement components C3 and/or C4 (21\% of cases, especially in IgG4-related renal disease) and rheumatoid factor and a low anti-nuclear antibody index [30].

Medical imaging facilitates the diagnosis, yet it is not specific enough to differentiate between IgG4-RD and inflammation or a hyperplastic process. It is believed that the main role is played here by computed tomography, magnetic resonance imaging [26] and, above all, FDG PET-CT [31].

\section{Treatment methods}

Beginning the treatment immediately after the diagnosis of IgG4-RD is not always necessary. In the case of oligosymptomatic forms of the disease, with a mild course, the treatment may be postponed on condition that the patient is carefully monitored ("watchful waiting").

Until now there have been no unified directives established regarding the treatment of IgG4-RD. Glucocorticoid administration is the first-choice therapy in patients with an active, previously untreated form of IgG4-RD. According to the directives from international guidelines, the common initial dose of prednisone $(0.6 \mathrm{mg} / \mathrm{kg}$ body weight/ day, 30-40 mg/day) should be administered for 2-4 weeks, and then gradually reduced. One scenario is to taper the daily dosage by $10 \mathrm{mg}$ every 2 weeks until a daily dosage of $20 \mathrm{mg}$ is reached. After a short time (e.g., 2 weeks) of treatment at $20 \mathrm{mg} / \mathrm{day}$, the tapering should resume by decreasing the daily dosage by $5 \mathrm{mg}$ every 2 weeks. The patient should terminate the treatment after a period of 3-6 months. However, some Japanese findings suggest that administration of small prednisone doses $(2.5-5 \mathrm{mg} /$ day) may be continued up to three years. In remittent and treatment-resistant forms of the disease studies have shown the efficacy of certain immunosuppressive drugs (azathioprine, 6-mercaptopurine, methotrexate, tacrolimus, cyclophosphamide) and biological compounds (rituximab) [27].

Treatment of IgG4-related endocrinopathies is believed to be the same as in patients with IgG4-related disease, but their use in management needs to be evaluated.

\section{Risk of neoplastic disease}

According to some findings, the risk of cancer increases threefold in patients with IgG4-RD in comparison to the general population [32]. The medical literature describes cases of non-Hodgkin lymphoma as well as lung carcinoma, pancreatic cancer, stomach cancer, bile duct cancer, kidney cancer, breast cancer, tongue cancer, melanoma and acute myeloid leukemia [33]. There have been reported single cases of concurrence of Riedel's thyroiditis and thyroid cancer [34].

\section{Thyroid disorders associated with lgG4-related disease}

The concept of IgG4-related thyroid disease was first proposed in 2011. It was then observed that $19 \%$ (22 out of 114) of patients with IgG4-related diseases present clinical markers of hypothyroidism. It was observed that glucocorticoid administration in 10 patients with clinically symptomatic hypothyroidism resulted in a statistically significant improvement in their hormonal parameters [35].

So far, on the basis of existing medical publications, thyroid disorders associated with IgG4-RD may be divided into Riedel's thyroiditis (RT), IgG4-related Hashimoto thyroiditis, (IgG4-RHD), fibrous variant of Hashimoto thyroiditis (FVHT), and Graves' disease related to elevated levels of IgG4 [36].

Riedel's thyroiditis is a very rare disease, with a frequency of 1.06/100 000 in the whole population [35]. Contrary to IgG4-RD, Riedel's thyroiditis occurs more frequently in women than in men (3:1). The peak age of incidence is between 30 and 60 years. The etiopathology of the disease has not been fully clarified. Most common in clinical manifestation is massive fibrosis of the thyroid gland and the surrounding tissues [37]. In clinical examination the thyroid is enlarged, painless and extremely hard, fixed to adjacent structures. Both in clinical examination and during surgery, it is difficult to distinguish between Riedel's thyroiditis and thyroid anaplastic cancer or lymphoma. Moreover, patients may have symptoms of tracheal and esophageal pressure (dysphonia, dyspnea, stridor, dysphagia), monolateral or bilateral paralysis of the recurrent laryngeal nerve, hypoparathyroidism, Horner's syndrome, or superior vena cava syndrome. A few dozen percent of patients present high levels of anti-thyroid antibodies, yet their role in pathogenesis of the disease remains unknown [38]. Schwaegerle et al. observed elevated levels of anti-thyroid antibodies in $67 \%$ of patients with RT. Upon diagnosis most patients are euthyroid, and approximately one third of them develop hypoactivity of the gland [39]. The microscopic image in RT presents similar results as the typical image of IgG4-RD and serves as the basis for the diagnostic and therapeutic process. In 2010 Dahlgren et al. reported cases of 3 patients with RT, using immunohistochemical tests to demonstrate the presence of IgG4+ cell infiltrations in the thyroid gland [40]. On the basis of later research RT has been included in the IgG4-RD spectrum [1]. 
The choice of treatment in patients with Riedel's thyroiditis depends primarily on the stage of the disease. In case of hypothyroidism, among the drugs, along with L-thyroxine supplementation, an important role is played by glucocorticoids. Glucocorticoid starting doses range widely from 15 to $100 \mathrm{mg} /$ day, and are generally reduced slowly over weeks to months. Remission rates after glucocorticoid usage are unclear, but relapse can occur during dose reduction. Nonsmokers respond better than smokers [41].

Tamoxifen is the second-line agent which has been used successfully in patients who relapse on glucocorticoids. Doses of 10-20 mg of tamoxifen either in combination with continued glucocorticoid therapy or as a monotherapy have been reported to be successful, especially in symptom control and reduction of pathological tissue mass [42].

In treatment-resistant patients mycophenolate mofetil and rituximab are of great importance for RT treatment $[43,44]$.

Hashimoto disease (HD) is the most common autoimmune disease and at the same time the most common cause of hypothyroidism in iodine-sufficient areas. The incidence of this disease increases with age (over $17 \%$ above the age of 60 ) and is much higher in women than in men $(7: 1)$. Most common in a typical clinical manifestation are: painless, diffuse goiter of increased density, elevated levels of anti-thyroid antibodies and hormonal markers of hypothyroidism, especially at the more advanced stage of the disease. There are a few clinical variants of HD [45], one of them being IgG4-related Hashimoto thyroiditis (IgG4RHD). According to Chinese studies, the incidence of IgG4-RHD among patients with HD is $22.64 \%$ (12 out of 53 cases) [46]. Similarly to IgG4-RD, the medical manifestation of this disease is characterized by fibrosis with lymphoplasmacytic infiltrations and follicular cell degeneration, which rapidly lead to hypothyroidism [47, 48]. Additional examination shows high levels of anti-thyroid antibodies and diffuse hypoechogenic lesions in ultrasonographic imaging [49].

Recognized since 1974, fibrous variant of Hashimoto's thyroiditis (FVHT) appears to be included in IgG4-RHD; however, there are no unified findings distinguishing these two disease entities [50]. Contrary to RT, there is so far no clinical proof of the efficacy of glucocorticoids in HD treatment [51]. Table III presents clinical markers distinguishing RT from FVHT [52].

During the research into the role of IgG4 in the diagnostics of autoimmune thyroid diseases, it has been observed that it may be used as a potential marker of disease activity. Elevated lgG4 levels in serum are reported in up to one third of patients with HD. This group develops a more aggressive course of the disease, requiring higher doses of L-thyroxine [53], although there are publications which contradict this observation [54].

Similar correlations have been searched for in patients with Graves' disease, yet it has been observed that only a small group of patients (6.4\%) are "IgG4-positive". However, the group's unique characteristics, such as proneness to hypothyroidism following the antithyroid therapy and greater tendency to develop Graves' ophthalmopathy, necessitate conducting further research in this field [55].

On the basis of currently available findings, it seems that lgG4-related thyroid disease must be considered in patients with a clinically aggressive form of the disease, and an immunohistochemical test with lgG4/lgG assessment should be performed in all of those patients.

\section{IgG4-related pituitary disease}

Primary inflammatory conditions of the pituitary gland are relatively rare, accounting for only $0.5 \%$

Table III. Clinical markers for distinguishing between Riedel's thyroiditis and fibrous variant of Hashimoto disease

\begin{tabular}{|lcc|}
\hline Marker & Riedel's thyroiditis & $\begin{array}{c}\text { Hashimoto disease - fibrous } \\
\text { variant }\end{array}$ \\
\hline Anti-thyroid antibody index & Moderately elevated & Significantly elevated \\
\hline Presence of normal thyroid tissue & Yes, clearly separate & No, diffuse lesions \\
\hline Vasculitis & Yes & No \\
\hline Fibrosis outside of the thyroid gland & Yes & No \\
\hline Hurthle cells & No & Mainly IgG, IgA (<15\%) \\
\hline Presence of antibodies in serum & IgA (47\%) & Yes \\
\hline Concurrence of autoimmune disease & Yes & Hypoechogenic \\
\hline Ultrasound image & Hypoechogenic & Increased \\
\hline Blood flow in Doppler examination & Reduced & (n) \\
\hline
\end{tabular}


of all causes of hypoactivity of this gland [56]. Until quite recently, there were four basic forms of histopathological inflammation: lymphocytic (most frequent), granulomatous, xanthomatous and necrotizing. One of the newly discovered forms is IgG4-related hypophysitis (IgG4-RH), which accounts for merely $1.5 \%$ of systemic cases of IgG4$\mathrm{RD}$ [2]. The first publications regarding this disease entity appeared in 2004 and described a case of a 66-year-old woman with numerous pseudotumorous lesions in the salivary glands, pancreas and peritoneal cavity, with concurrent secondary hypothyroidism and sella turcica enlargement in tomographic imaging of the pituitary gland [57]. Further studies systematized the clinical and radiological manifestation of this condition. Similarly to IgG4-RD, the incidence is higher in men than in women, and the mean age of onset is 64 years. General weakness, headaches and vision disorders are predominant in clinical examination. Other symptoms include polyuria, weight loss, high body temperature and decreased libido. Also observed are various degrees of hypoactivity of the anterior lobe of the pituitary gland and central diabetes insipidus. Complete pituitary hypoactivity occurs in $52.4 \%$ of the described cases, and as many as $75 \%$ of patients develop antidiuretic hormone deficiency [58]. Magnetic resonance imaging of the pituitary is not very specific, mostly showing edema or pseudotumorous enlargement of the gland. A "bright spot" in the posterior lobe may be observed in patients with central diabetes insipidus. Similar lesions also occur in other inflammatory conditions of the pituitary gland (e.g. lymphocytic inflammation), as well as in sarcoidosis, tuberculosis and Wegener's granulomatosis [59].

In 2011 Leporati et al. proposed three diagnostic criteria of IgG4-related pituitary disease, the fulfillment of which is sufficient for establishing the diagnosis. The first criterion requires a microscopic examination of the pituitary gland, with confirmed presence of mononuclear cell infiltration with over 10 lgG 4 cells in the field of vision. The second criterion is atypical manifestation of pituitary gland inflammation visible in MRI and confirmed infiltrations located elsewhere. The last criterion is the concurrence of elevated IgG4 levels in serum, manifestation of pituitary inflammation in $\mathrm{MRI}$ and rapid symptom remission following glucocorticoid administration [60].

The diagnostics of IgG4-related pituitary disease undoubtedly poses a serious challenge for clinicians. The microscopic image and the result of immunohistochemical examination may not differ significantly from other variants of pituitary inflammatory conditions. Lymphocytic infiltrations of IgG4 cells have also been described in connection with other diseases [61]. A diagnosis for IgG4-
$\mathrm{RH}$ should be considered in patients who develop meningitis or have a relapse induced by reduction of the glucocorticoid dose [62].

The typical therapy for IgG4-related hypophysitis is undefined. It is noteworthy that there are clinical cases in which high clinical efficacy of small glucocorticoid doses is reported from the beginning of treatment $[63,64]$.

\section{IgG4-related disease versus focal adrenal lesions}

Focal adrenal lesions regarded as IgG4-related are extremely rare. The only example of this condition confirmed so far is calcifying fibrous tumor that belongs to a group of benign mesenchymal cancers, which usually develops in the stomach, lungs, mediastinum, peritoneum, and in approximately $3 \%$ of cases also the adrenal glands [65]. A classic example, described in 2016, is a case of a 32-year-old patient with a lesion in the left adrenal gland accidentally detected during an ultrasound examination. The medical picture was asymptomatic and hormonal test results were within the reference range [66].

\section{IgG4-related ophthalmic disease}

Graves' orbitopathy is a frequent clinical problem in endocrine practice, affecting on average $25 \%$ of patients with Graves' disease [38]. In differential diagnosis of this condition a range of diseases has to be taken into consideration, with IgG4-related ophthalmic disease recently classified as one of them [67].

The first findings regarding IgG4-RD with infection of the orbital soft tissues were described in 2011 in Italy, when Fonte et al. presented a case of a patient with autoimmune pancreatitis and Graves' orbitopathy, with attendant high IgG4 levels in serum and euthyroid. The clinical course of the disease was similar to an active, acute or moderate form of Graves' ophthalmopathy. A good response to intravenous glucocorticoid administration and radiotherapy of the orbital cavities was observed [68]. On the basis of further studies the term "IgG4-related ophthalmopathy" was proposed [1].

In the clinical picture the disease is usually bilateral and is characterized by a mild course. Pain sensation in the eyes or visual field disorders do not occur frequently. Most common in clinical examination are chronic eyelid edema and exophthalmos, with uninhibited eye movement. There is a possibility of infection of the lacrimal gland (70\%), orbital bone structure, ophthalmic nerve or a branch of the trigeminal nerve (V1 and V2). Extraocular muscles are extended in one third of the patients. The most common location is the lat- 
eral rectus muscle [69-71]. Sometimes there can be observed symptoms resulting from infections of other organs such as submandibular glands (29\%), lymph nodes (14\%), pancreas (5\%) and bile ducts (5\%) [71-74]. Similarly to other forms of IgG4-related diseases, a histopathological examination is the basis for the diagnostic process. In the microscopic image lymphoplasmacytic and eosinophilic infiltrations prevail, whereas vein thrombosis is very rare [74]. The core of the therapy is glucocorticoids, which in approximately $70 \%$ of patients should be administered longterm in monotherapy or in conjunction with other immunosuppressive drugs. Biopharmaceuticals such as rituximab or bortezomib are suitable for patients with a treatment-resistant form of the disease $[27,71]$. The recommended dose of rituximab is two pulses of $1.0 \mathrm{~g}$ with a 2-week interval. Patients may require more than one cycle of rituximab, or may experience relapse after 6 months when peripheral B-cells are re-populated [75].

A diagnosis of IgG4-RD should be considered in the differential diagnosis of orbitopathy of unclear etiology, especially treatment-resistant and in cases of concurrent symptoms in other systems.

\section{Diabetes in the course of autoimmune pancreatitis}

Autoimmune pancreatitis is a syndrome consisting of general symptoms, obstructive jaundice and often concurrent carbohydrate imbalance. There are two types of AIP. Type 1 AIP (IgG4-related pancreatitis) is more prevalent in Japan and Korea, whereas type 2 AIP, with granulocytic epithelial lesions, is more commonly observed in Europe and the United States [76].

AIP-related diabetes may occur at every stage of the disease. However, in more than $50 \%$ of patients it is reported at the moment of the AIP diagnosis. It is remarkable that the symptoms of AIP-related hyperglycemia may completely disappear following glucocorticoid administration [77]. Around $60 \%$ of cases of diabetes mellitus associated with AIP are responsive to steroids in the short and long term [78]. Yamada el al. described a case of a 79-year-old woman whose diabetes was the only AIP manifestation, and glycemic balance after the first month of steroid therapy was maintained without hypoglycemic drug administration [79]. In the majority of cases, "IgG4-related diabetes" is treated with insulin therapy and its course depends on how advanced the pancreatic atrophy is [80-83].

\section{Conclusions}

IgG4-RD is a new, multidisciplinary condition, which poses a challenge to clinicians. The scope of this phenomenon and its role in contemporary diagnostics appear to be a subject which truly deserves further research as well as medical experiments. Thyroid and pituitary gland diseases constitute the highest percentage of IgG4-related endocrinopathies.

\section{Conflict of interest}

The authors declare no conflict of interest.

\section{References}

1. Stone JH, Khosroshahi A, Deshpande V, et al. Recommendation for the Nomenclature of IgG4-Related disease and its individual organ system manifestation. Arthritis Rheum 2012; 64: 3061-7.

2. Brito-Zerón P, Ramos-Casals M, Bosch X, Stone JH. The clinical spectrum of IgG4-related disease. Autoimmun Rev 2014; 13: 1203-10.

3. Brito-Zerón P, Bosch X, Ramos-Casals M, Stone JH. IgG4-related disease: advances in the diagnosis and treatment. Best Pract Res Clin Rheumatol 2016; 30: 261-78.

4. Umehara H, Okazaki K, Masaki Y, et al. Comprehensive diagnostic criteria for IgG4-related disease Mod Rheumatol 2012; 22: 21-30.

5. Kamisawa T, Funata N, Hayashi Y, et al. A new clinicopathological entity of IgG4-related autoimmune disease. J Gastroenterol 2003; 38: 982-4.

6. Wallace ZS, Deshpande V, Mattoo H, et al. IgG4-related disease: baseline clinical and laboratory features in 125 patients with biopsy-proven disease. Arthritis Rheumatol 2015; 67: 2466-75.

7. Katsura M, Mori H, Kunimatsu A, et al. Radiological features of IgG4-RD in the head, neck and brain. Neuroradiology 2012; 54: 873-82.

8. Chen H, Lin W, Wang Q, et al. IgG4-related disease in Chinese cohort: prospective study. Scand I Rheumatol 2014; 43: 70-4.

9. Batu ED, Arici ZS, Orhan D, Kiratli H, Özen S. Immunoglobulin G4-related orbital disease: pediatric case. Clin Exp Rheumatol 2015; 33: 409-10.

10. Corujeira S, Ferraz C, Nunes T, Fonseca E, Vaz LG. Severe IgG4-related disease in young child: a diagnosis challenge. Case Rep Pediatr 2015; 2015: 140753.

11. Aalberse RC, Schuurman J. IgG4 breaking the rules. Immunology 2002; 105: 9-19.

12. Aalberse RC, Stapel SO, Schuurman J, Rispens T. Immunoglobulin G4: an odd antibody. Clin Exp Allergy 2009; 39: 469-77.

13. Culver EL, Vermeulen E, Makuch M, et al. Increased IgG4 responses to multiple food and animal antigens indicate a polyclonal expansion and differentiation of pre-existing B cells in IgG4-related disease. Ann Rheum Dis 2015; 74: 944-7.

14. Vijay HM, Perelmutter L. Inhibition of reagin-mediated PCA reactions in monkeys and histamine release from human leukocytes by human IgG4 subclass. Int Arch Allergy Appl Immunol 1977; 53: 78-87.

15. Gwynn CM, Ingram J, Almousawi T, Stanworth DR. Bronchial provocation tests in atopic patients with allergen specific IgG4 antibodies. Lancet 1982; 1: 254-6.

16. van der Giessen M, Homan WL, van Kernbeek G, Aalberse RC, Dieges PH. Subclass typing of IgG antibodies formed by grass pollen allergic patients during immunotherapy. Int Arch Allergy Appl Immunol 1976; 50: $625-40$. 
17. Okazaki K, Uchida K, Ohana M, et al. Autoimmune-related pancreatitis is associated with autoantibodies and a Th1/Th2-type cellular immune response. Gastroenterology 2000; 118: 573-81.

18. Frulloni L, Lunardi C, Simone R, et al. Identification of a novel antibody associated with autoimmune pancreatitis. N Engl J Med 2009; 361: 2135-42.

19. Du H, Shi L, Chen P, et al. Prohibitin is involved in patients with IgG4 related disease. PLoS One 2015; 10 e0125331

20. Guarneri F, Guarneri C, Benvenga S. Helicobacter pylor and autoimmune pancreatitis: role of carbonic anhydrase via molecular mimicry? J Cell Mol Med 2005; 9: 741-4.

21. Zen Y, Nakanuma Y. Patogenesis of IgG4-related disease. Curr Opin Rheumatol 2011; 23: 114-8.

22. Della Torre E, Mattoo H, Mahajan VS, Carruthers M, Pillai S, Stone JH. Prevalence of atopy, eosinophilia, and IgE elevation in IgG4-related disease. Allergy 2014; 69 269-72.

23. Furukawa S, Moriyama M, Tanaka A, et al. Preferential M2 macrophages contribute to fibrosis in IgG4-related dacryoadenitis and sialoadenitis, so-called Mikulicz's disease. Clin Immunol 2015; 156: 9-18.

24. Wallace ZS, Mattoo $\mathrm{H}$, Carruthers $M$, et al. Plasmablasts as a biomarker for lgG4-related disease, independent of serum IgG4 concentrations. Ann Rheum Dis 2015; 74 190-5.

25. Umehara H, Nakajima A, Nakamura T, et al. IgG4-related disease and its pathogenesis - cross-talk between innate and acquired immunity. Int Immunol 2014; 26 : 585-95.

26. Stone JH, Brito-Zerón P, Bosch X, Ramos-Casals M. Diagnostic approach to the complexity of IgG4-related dis ease. Mayo Clin Prac 2015; 90: 927-39.

27. Khosroshahi A, Wallace ZS, Crowe JL, et al.; Second International Symposium on IgG4-Related Disease. International Consensus Guidance Statement on the management and treatment of IgG4-related disease. Arthritis Rheumatol 2015; 67: 1688-99.

28. Deshpande V, Zen Y, Chan JK, et al. Consensus state ment on the pathology of IgG4-related disease. Mod Pathol 2012; 25: 1181-92.

29. Yamamoto $M$, Yajima $H$, Takahashi $H$, et al. Everyday clinical practice in IgG4-related dacryoadenitis and/or sialadenitis: results from the SMART database. Mod Rheumatol 2015; 25: 199-204.

30. Kazuhiro K, Hajime Y, Tsugumitsu K, et al. Screening for IgG4-type anti-nuclear antibodies in IgG4-related disease. BMC Musculoskelet Disord 2015; 16: 129.

31. Lauwyck J, Piette Y, Van Walleghem L, De Geeter F. IgG4-related disease: the utility of (18)F-FDG PET/CT in diagnosis and treatment. Hell J Nucl Med 2015; 18 Suppl 1: 155-9.

32. Yamamoto M, Takahashi H, Tabeya T, et al. Risk of malignancies in IgG4-related disease. Mod Rheumatol 2012; 22: 414-8.

33. Hirano K, Tada M, Sasahira N, et al. Incidence of ma lignancies in patients with IgG4-related disease. Intern Med 2014; 53: 171-6.

34. Hao SP, Chen JF, Yen KC. Riedel's thyroiditis associated with follicular carcinoma. Eur Arch Otorhinolaryngo 1999; 256: 470-2

35. Watanabe T, Maruyama M, Ito T, et al. Clinical features of a new disease concept, IgG4-related thyroiditis. Scand Rheumatol 2013; 42: 325-30.

36. Kottahachchi D, Topliss DJ. Immunoglobulin G4-related thyroid diseases. Eur Thyroid J 2016; 5: 231-9.
37. Wang CJ, Wu TJ, Lee CT, Huang SM. A misdiagnosed Riedel's thyroiditis successfully treated by thyroidectomy and tamoxifen. J Formos Med Assoc 2012; 111: 719-23.

38. Zgliczyński W. Wielka interna: endokrynologia. Medical Tribune Polska, Warsaw 2011; 291-2.

39. Schwaegerle SM, Bauer TW, Esselstyn CB. Riedel's thyroiditis. Am J Clin Pathol 1988; 90: 715-22.

40. Dahlgren M, Khosroshahi A, Nielsen GP, Deshpande V, Stone JH. Riedel's thyroiditis and multifocal fibrosclerosis are part of the IgG4-related systemic disease spectrum. Arthritis Care Res (Hoboken) 2010; 62: 1312-8.

41. Fatourechi MM, Hay ID, Mclver B, Sebo TJ, Fatourechi V. Invasive fibrous thyroiditis (Riedel thyroiditis): the Mayo Clinic experience, 1976-2008. Thyroid 2011; 21: 765-72.

42. Kottahachchi D, Topliss DJ. Immunoglobulin G4-related thyroid diseases. Eur Thyroid J 2016; 5: 231-9.

43. Levy JM, Hasney CP, Friedlander PL, Kandil E, Occhipinti EA, Kahn MJ. Combined mycophenolate mofetil and prednisone therapy in tamoxifen- and prednisone-resistant Riedel's thyroiditis. Thyroid 2010; 20: 105-7.

44. Carruthers MN, Topazian MD, Khosroshahi A, et al. Rituximab for IgG4-related disease: a prospective, openlabel trial. Ann Rheum Dis 2015; 74: 1171-7.

45. Hiromatsu Y, Satoh H, Amino N. Hashimoto's thyroiditis: history and future outlook. Hormones (Athens) 2013; 12: $12-8$.

46. Zhang J, Zhao L, Gao Y, et al. A classification of Hashimoto's thyroiditis based on immunohistochemistry for IgG4 and IgG. Thyroid 2014; 24: 364-70.

47. Li Y, Bai Y, Liu Z, et al. Immunohistochemistry of IgG4 can help subclassify Hashimoto's autoimmune thyroiditis. Pathol Int 2009; 59: 636-41.

48. Deshpande V, Huck A, Ooi E, Stone JH, Faquin WC, Nielsen GP. Fibrosing variant of Hashimoto thyroiditis is an IgG4 related disease. J Clin Pathol 2012; 65: 725-8.

49. Li Y, Nishihara E, Hirokawa M, Taniguchi E, Miyauchi A, Kakudo K. Distinct clinical, serological, and sonographic characteristics of Hashimoto's thyroiditis based with and without IgG4-positive plasma cells. J Clin Endocrinol Metab 2010; 95: 1309-17.

50. Katz SM, Vickery AL Jr. The fibrous variant of Hashimoto's thyroiditis. Hum Pathol 1974; 5: 161-70.

51. Dutta D, Ahuja A, Selvan C. Immunoglobulin G4 related thyroid disorders: diagnostic challenges and clinical outcomes. Endokrynol Pol 2016; 67: 520-4.

52. Hennessey JV. Clinical review: Riedel's thyroiditis: a clinical review. J Clin Endocrinol Metab 2011; 96: 3031-41.

53. Popławska-Kita A, Kościuszko-Zdrodowska M, Siewko K, et al. High serum lgG4 concentrations in patients with Hashimoto's thyroiditis. Int J Endocrinol 2015; 2015: 706843.

54. Takeshima K, Ariyasu $\mathrm{H}$, Inaba $\mathrm{H}$, et al. Distribution of serum immunoglobulin G4 levels in Hashimoto's thyroiditis and clinical features of Hashimoto's thyroiditis with elevated serum immunoglobulin G4 levels. Endocin J 2015; 62: 711-7.

55. Bozkirli E, Bakiner OS, Ersozlu Bozkirli ED, et al. Serum immunoglobulin G4 levels are elevated in patients with Graves' ophthalmopathy. Clin Endocrinol (Oxf) 2015; 83: 962-7.

56. Howlett TA, Levy MJ, Robertson IJ. How reliably can autoimmune hypophysitis be diagnosed without pituitary biopsy. Clin Endocrinol (Oxf) 2010; 73: 18-21.

57. van der Vliet HJ, Perenboom RM. Multiple pseudotumors in IgG4-associated multifocal systemic fibrosis. Ann Intern Med 2004; 141: 896-7.

58. Shikuma J, Kan K, Ito R, et al. Critical review of IgG4-related hypophysitis. Pituitary 2017; 20: 282-91. 
59. Shimatsu A, Oki Y, Fujisawa I, Sano T. Pituitary and stalk lesions (infundibulo-hypophysitis) associated with immunoglobulin G4-related systemic disease: an emerging clinical entity. Endocr J 2009; 56: 1033-41.

60. Leporati P, Landek-Salgado MA, Lupi I, Chiovato L, Caturegli P. IgG4-related hypophysitis: a new addition to the hypophysitis spectrum. J Clin Endocrinol Metab 2011; 96: 1971-80.

61. Nishioka H, Shibyya M, Haraoka J. Immunohistochemical study for IgG4-positive plasmacytes in pituitary inflammatory lesions. Endocr Pathol 2010; 21: 236-41.

62. Nqaosuwan K, Trongwongsa T, Shuangshotu S. Clinical course of IgG4-related hypophysitis presenting with focal seizure and relapsing lymphocytic hypophysitis. BMC Endocr Disord 2015; 15: 64.

63. Harano Y, Honda K, Akiyama Y, Kotajima L, Arioka H. A case of IgG4-related hypophysitis presented with hypopituitarism and diabetes insipidus. Clin Med Insights Case Rep 2015; 8: 23-6.

64. Hori M, Makita N, Andoh T, et al. Long-term clinical course of IgG4-related systemic disease accompanied by hypophysitis. Endocr J 2010; 57: 485-92.

65. Chorti A, Papavtamidis TS, Michalopoulos A. Calcifying fibrous tumor review of 157 patients reported in international literature. Medicine (Baltimore) 2016; 95: e3690.

66. Wu T, Zhu P, Duan X, Yang X, Lu D. Calcifying fibrous pseudotumor of the adrenal gland: a rare case report. Mol Clin Oncol 2016; 5: 252-4.

67. Bartalena L, Chiovato L. Graves'-like orbitopathy: do not forget IgG4-related disease. J Endocrinol Invest 2014; 37: 1233-5.

68. Fonte R, Pirali B, Caramia V, et al. Graves'-like orbitopathy in a patient with chronic autoimmune pancreatitis. Thyroid 2011; 21: 1389-92.

69. Tiegs-Heiden CA, Eckel LJ, Hunt CH, et al. Immunoglobulin G4-related disease of the orbit: imaging features in 27 patients. AJNR Am J Neuroradiol 2014; 35: 1393-7.

70. Moritani KT. Orbital IgG4-related disease: clinical features and diagnosis. ISRN Rheumatol 2012; 2012 412896.

71. Ebbo M, Patient M, Grados A, et al. Ophthalmic manifestations in IgG4-related disease Clinical presentation and response to treatment in a French case-series. Medicine (Baltimore) 2017; 96: e6205.

72. Kubota T, Moritani S, Katayama M, Tarasaki H. Ocular adnexal IgG4-related lymphoplasmacytic infiltrative disorder. Arch Ophthalmol 2010; 128: 577-84.

73. Plaza JA, Garrity JA, Dogan A, Ananthamurthy A, Witzig TE, Salomão DR. Orbital inflammation with IgG4-positive plasma cells: manifestation of IgG4 systemic disease. Arch Ophthalmol 2011; 129: 421-8.

74. Sato $\mathrm{Y}$, Ohshima $\mathrm{K}$, Ichimura $\mathrm{K}$, et al. Ocular adnexal IgG4-related disease has uniform clinicopathology. Pathol Int 2008; 58: 465-70.

75. Wallace ZS, Deshpande V, Stone JH. Ophthalmic manifestations of IgG4-related disease: single-center experience and literature review. Semin Arthritis Rheum 2014; 43: 806-17.

76. Kanno A, Masamune A, Okazaki K, et al.; Research Committee of Intractable Diseases of the Pancreas. Nationwide epidemiological survey of autoimmune pancreatitis in Japan in 2011. Pancreas 2015; 44: 535-9.

77. Okazaki K, Kawa S, Kamisawa T, et al.; Working Committee of the Japan Pancreas Society and the Research Committee for Intractable Pancreatic Disease supported by the Ministry of Health, Labour and Welfare of Japan.
Amendment ofthe Japanese Consensus Guidelines fo Autoimmune Pancreatitis, 2013 I. Concept and diagnosis of autoimmune pancreatitis. J Gastroenterol 2014, 49: 567-88.

78. Miyamoto Y, Kamisawa T, Tabata T, et al. Short and long term outcomes of diabetes mellitus in patients with au toimmune pancreatitis after steroid therapy. Gut Liver 2012; 6: 501-4.

79. Yamada T, Hiraoka E, Miyazaki T, Sato J, Ban N. Diabetes as first manifestation of autoimmune pancreatitis. Am Med Sci 2017; 353: 498-9.

80. Masuda A, Shimomi H, Matsuda T, et al. The relationship between pancreatic atrophy after steroid therapy and diabetes mellitus in patients with autoimmune pancreatitis. Pancreatology 2014; 14: 361-5.

81. Ito T, Nakamura T, Fujumori N, et al. Characteristic of pancreatic diabetes in patiens with autoimmune pancreatitis. J Dig Dis 2011; 12: 210-6.

82. Inoue D, Yoshida K, Yoneda N, et al. IgG4-related disease: data of 235 consecutive patients. Medicine (Baltimore) 2015; 94: e680.

83. Ebbo M, Daniel L, Pavic M, et al. IgG4-related systemic disease: features and treatment response in a French cohort: results of a multicenter registry. Medicine (Baltimore) 2012; 91: 49-56. 